\title{
Evaluation of adjuvant psychological therapy in patients with testicular cancer: randomised controlled trial
}

\author{
C Moynihan, J M Bliss, J Davidson, L Burchell, A Horwich
}

\begin{abstract}
Objective: To determine the efficacy of adjuvant psychological therapy in patients with testicular cancer and to compare the characteristics and psychosocial outcomes of men who agreed to participate with those who declined to participate in a randomised trial of psychological intervention.

Design: Newly diagnosed patients were asked to participate in a randomised trial of psychological support compared with standard medical care. Participants and non-participants completed self assessment questionnaires at baseline and at 2, 4 and 12 months.
\end{abstract}

Setting: Testicular Tumour Unit of the Royal Marsden Hospital.

Subjects: 73 of $184(40 \%)$ eligible patients agreed to enter the randomised trial (participants) and 81 (44\%) declined to participate but agreed to complete further assessments (non-participants). 30 patients wanted no further contact with the researchers.

Outcome measures: Hospital anxiety and depression scale, psychosocial adjustment to illness scale, Rotterdam symptom checklist, mental adjustment to cancer scale. Only scores on the hospital anxiety and depression scale are reported for evaluating treatment efficacy.

Results: 111 of $184(60 \%)$ eligible men declined to participate in the trial. Patients with stage I disease were most likely to refuse to participate. A patient was less likely to participate if he had low volume disease and was receiving no further treatment. Likelihood of participation was associated with stage of disease and with type of primary treatment $(\mathrm{P}<0.001$ for heterogeneity). Patients with early stage disease $(\mathrm{P}<0.001)$ and fewer physical symptoms $(\mathrm{P}<0.001)$ were less likely to participate. Psychosocial factors associated with participation included anxious preoccupation regarding disease $(\mathrm{P}=0.01)$. There were no differences in outcome between participants and non-participants during follow up. Patients seemed to gain little benefit from adjuvant psychological therapy. At 2 months change from baseline favoured the treated group in the anxiety subscale (mean difference between groups -1.41 (95\% confidence interval -2.86 to 0.03$)$ ). This was not sustained when adjusted for factors related to the disease. By 12 months change from baseline seemed to favour the control group (mean difference between groups 1.66 ( -0.18 to 3.50$)$ ).

Conclusions: Patients with testicular cancer seem to have considerable coping abilities. Those who declined to participate in the trial differed from those who participated. Those who agreed to participate may comprise the clinical group who perceive a need for psychological support. No evidence was found to indicate a need for routinely offering adjuvant psychological therapy.

\section{Introduction}

Counselling and psychotherapy are advocated for and used in various medical settings, ${ }^{1}$ so these interventions need to be fully evaluated. In oncology, results of methodologically sound studies suggest both positive $\mathrm{e}^{2-5}$ and negative outcomes ${ }^{67}$ of counselling, although the clinical importance or magnitude of any effect cannot always be extrapolated. Neither is it possible to evaluate the consistency of results between studies since a variety of measures are used and results often do not include any statement of precision, such as confidence intervals. To date, no study of patients with cancer has evaluated a psychological intervention in a group of young male patients or in a group with a disease with an excellent prognosis.

Many patients with testicular cancer experience psychosocial morbidity after treatment has ended. ${ }^{8}$ There is evidence that few in this group seek psychotherapeutic help, ${ }^{910}$ although in one study, when a hypothetical offer was made, 62/102 (61\%) stated that they needed counselling. ${ }^{9}$ These patients also preferred individual counselling to begin soon after diagnosis or at the start of treatment, and indicated that the sex of the therapist was unimportant. Patients' perceived need for counselling was not associated with concurrent psychological morbidity.

Those who decline entry into a trial of psychotherapeutic intervention and thus the offer of psychological support are seldom assessed. This makes it difficult to understand the psychosocial and medical characteristics that influence a patient's decision to attend counselling and how these characteristics may affect the outcome of counselling. ${ }^{11}$

Since men are regarded as having a problem focused coping strategy the preferred psychotherapeutic intervention for men is one that focuses on regaining control. ${ }^{12}$ Adjuvant psychological therapy, which uses both cognitive and behavioural approaches, includes strategies such as problem solving and regaining control ${ }^{13}$ and has long term benefits for patients with cancer. $^{5}$

We evaluated the efficacy of adjuvant psychological therapy in a randomised controlled trial, and investigated the medical and psychosocial characteristics and psychosocial outcome in those who agreed and those who declined to participate.

\section{Subjects and methods}

\section{Patient population and trial procedure}

Newly diagnosed non-suicidal patients with testicular cancer referred to the Royal Marsden Hospital between 1988 and 1990 were asked to participate in the study. Patients were eligible to participate if they were aged between 18 and 65 years, had had a unilateral orchidectomy, and had been advised of and agreed to a treatment plan. Randomisation to adjuvant psychological therapy or standard care was performed
Academic

Department of Radiotherapy and Oncology, Royal Marsden NHS Trust, Sutton, Surrey SM2 5PT

C Moynihan, medical sociologist L Burchell, research assistant A Horwich, professor of radiotherapy and oncology

Clinical Trials and Statistics Unit, Section of

Epidemiology,

Institute of Cance

Research, Sutton, Surrey SM2 5NG

J M Bliss,

statistician

J Davidson,

data manager

Correspondence to:

Ms Moynihan

clare@icr.ac.uk

BMJ 1998;316:429-35 
by an independent trials office. Patients who declined to participate were asked to complete psychosocial assessments using the same procedures as patients in the randomised trial (non-participants).

\section{Assessment procedures}

The researcher (CM) who coordinated the completion of assessments was blind to a patient's allocation within the trial but was aware of whether a patient had agreed to participate or not. Self report assessments were performed at baseline (before randomisation for patients participating in the trial) and at 2, 4, and 12 months. Patients completed the following validated self report questionnaires: the hospital anxiety and depression scale,$^{14}$ the mental adjustment to cancer scale, ${ }^{15}$ the psychosocial adjustment to illness scale, ${ }^{16}$ the Rotterdam symptom checklist, ${ }^{17}$ the emotional concealment subscale of the Brannon masculinity scale ${ }^{18}$ and the Rieker sexual adjustment scale. ${ }^{10}$

Another self report questionnaire (formulated by us) provided details of patients' perceptions of emotional or psychological illness and any drugs they had taken, including alternative medical remedies, within the time under review (from before diagnosis or since the previous assessment). Patients were asked their reasons for declining to participate in the trial. Demographic, social, and clinical information was drawn from patients' clinical notes. A patient's risk of suicide was established at baseline using a validated objective measure for assessment of patients with cancer. $^{19}$

Several outcome measures were used to provide descriptive information and to allow for comparison with other studies. A previous study of adjuvant psychological therapy showed that the hospital anxiety and depression scale was the most informative in detecting clinical effect $^{45}$; for this reason only

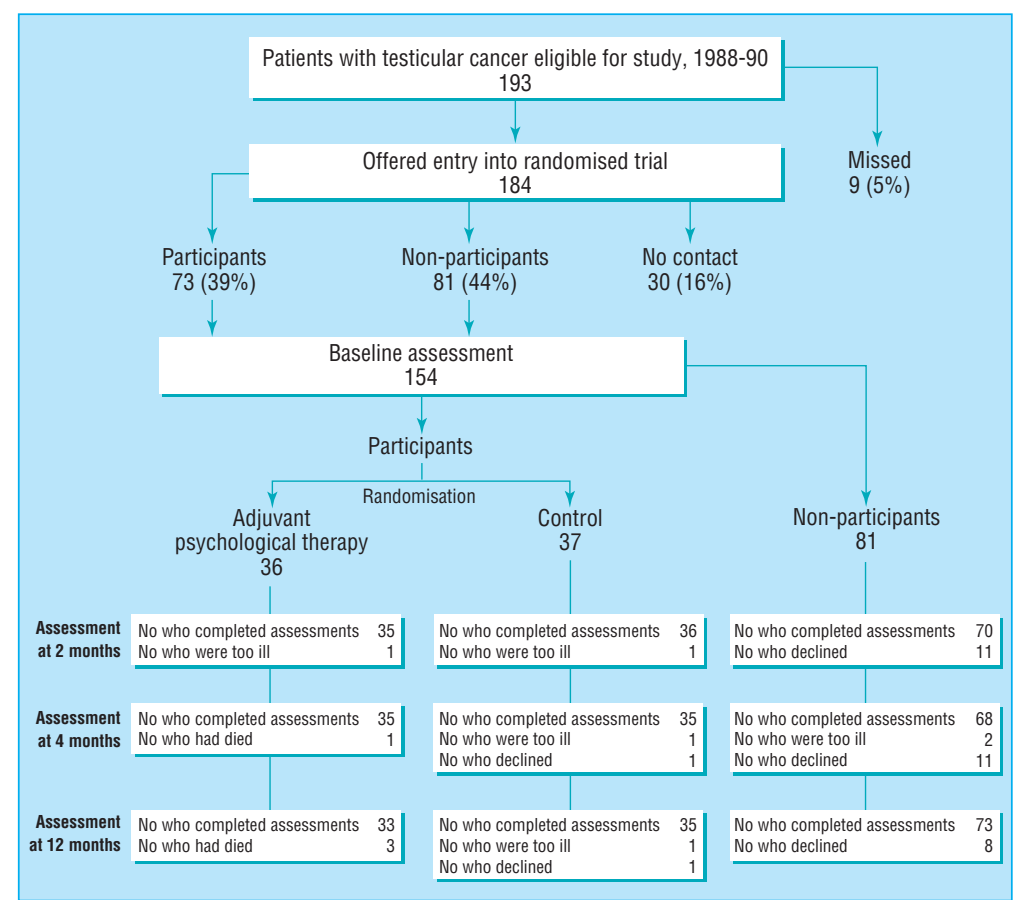

Design of randomised controlled trial of psychotherapeutic intervention $v$ standard medical care in patients with testicular cancer. Number of patients who completed self assessment questionnaires at baseline and 2, 4, and 12 months also shown
Table 1 Patients' reasons for declining to enter randomised controlled trial of adjuvant psychological therapy. Values are numbers (percentages)

\begin{tabular}{lcc} 
Reason* & $\begin{array}{c}\text { Non-participants } \\
(\mathbf{n}=\mathbf{8 1}) \boldsymbol{\dagger}\end{array}$ & $\begin{array}{c}\text { No contact } \\
(\mathbf{n}=\mathbf{3 0}) \neq\end{array}$ \\
\hline Would interfere with work & $18(22)$ & $5(17)$ \\
\hline Dislike of counselling & $12(15)$ & $4(13)$ \\
\hline Sufficient support already & $12(15)$ & $3(10)$ \\
\hline Sense of wellbeing & $51(63)$ & $16(53)$ \\
\hline Study site too far away & $6(7)$ & $13(43)$ \\
\hline Don't want to be reminded of illness & $4(5)$ & 0 \\
\hline Too ill & $2(2)$ & 0 \\
\hline
\end{tabular}

*Patients gave more than one reason for declining to participate.

†Patients who declined to participate but agreed to complete questionnaires. $\ddagger$ Patients who declined all further contact with researchers.

outcomes relating to this scale are reported. A detailed description of other outcome measures will be published elsewhere.

\section{Adjuvant psychological therapy}

Adjuvant psychological therapy is a cognitive and behavioural treatment programme designed specifically for patients with cancer; the techniques have been documented. ${ }^{4}{ }^{13}$ Six sessions, each lasting one hour, were scheduled between baseline assessment and evaluation at 8 weeks. Patients were informed that if resolution was reached the therapy would end early. Additional sessions were provided after 8 weeks when necessary. All sessions of adjuvant psychological therapy were tape recorded. The therapist was a state registered mental health nurse who was experienced in caring for patients with testicular cancer. The nurse was trained and supervised in adjuvant psychological therapy techniques by the hospital's department of psychological medicine.

\section{Statistical methods}

Data were analysed on an intention to treat basis-that is, comparisons included all patients who were randomised, according to allocated treatment, regardless of compliance. Similarly, comparisons between patients who agreed to participate and those who declined included all patients. Analysis of baseline data used a non-parametric approach because the data were not normally distributed. The outcome analysis uses the change in scores from baseline. Scores on the subscales were analysed separately at each point in time. Estimates of the change over time (within the group) and the treatment effect (between groups) were calculated. Although this reduced the skewness of the scores the non-normal distribution of some scores was considered because of the shape (kurtosis) of the distribution. Methods based on the Student's $t$ distribution are reasonably robust in such cases and were used to calculate a confidence interval for the mean change in scores. Since the power of the test of any hypothesis may be affected by having a non-normal distribution, however, formal comparison between the groups was complemented by a non-parametric approach using the Mann-Whitney U test.

Problems in interpretation may arise when several outcome measures are used because of the multiple comparisons. In this study no adjustment was made to individual $\mathrm{P}$ values to correct for multiple testing because the choice of denominator (the number of 
tests) was variable. Caution is therefore needed in the interpretation of individual $\mathrm{P}$ values.

The trial was designed to recruit 120 patients over 2 years. Recruitment was stopped after 21/2 years because of the low number of patients who agreed to participate. At that time no analysis of outcome data had been performed.

\section{Results}

\section{Patients}

A total of 193 patients were eligible to participate in the trial (figure). Of these, 184 patients were approached: $73(40 \%)$ agreed to participate (participants), 81 (44\%) declined to participate but agreed to complete assessments (non-participants), 30 (16\%) refused further contact with the researcher (no contact group). The remaining nine men were missed for administrative reasons. Table 1 shows the reasons why patients declined to participate.

\section{Data collection}

For participants, rates of questionnaire completion at 2, 4, and 12 months were 71/73 (97\%), 70/73 (96\%), and $68 / 73(93 \%)$; rates for non-participants were $70 / 81 \quad(86 \%), \quad 68 / 81 \quad(84 \%)$, and $73 / 81 \quad(90 \%)$ respectively. Altogether 10 assessments were missing for participants and 32 were missing for nonparticipants. Nine of the participants were unable to complete their assessments because of disease related factors. Only two of the non-participants were unable to complete their assessments because of disease related factors.

\section{Baseline characteristics of participants and non-participants}

Non-participants and those in the no contact category were similar to participants in demographic and social factors (table 2). A patient was less likely to participate if he had low volume disease and was receiving no further treatment. Likelihood of participation was associated with stage of disease $(\mathrm{P}<0.001$ for trend $)$ and with type of primary treatment $(\mathrm{P}<0.001$ for heterogeneity).

Baseline scores on psychosocial scales fell mainly within normal ranges (table 3). However, nonparticipants had less morbidity than participants, especially on the psychological $(\mathrm{P}<0.001)$ and physical $(\mathrm{P}<0.001)$ subscales of the Rotterdam symptom checklist. Non-participants were also more likely to deny their diagnosis of cancer $(\mathrm{P}=0.002)$.

There were no consistent differences between participants and non-participants in measures of psychosocial wellbeing over time. At one year the groups were remarkably similar; the only significant difference, in favour of participants, was observed in the physical symptom subscale of the Rotterdam symptom checklist (table 3). This effect was not seen at 2 months. This difference may be an artefact of the clinical characteristics of the participants. Psychosocial wellbeing seemed largely independent of histology, but scores on the depression subscale of the hospital anxiety and depression scale and the physical symptom subscale of the Rotterdam symptom checklist seemed to be influenced by stage of disease (both $\mathrm{P}<0.001$ ) and type of primary treatment $(\mathrm{P}=0.003, \mathrm{P}<0.001)$.
Table 2 Demographic, social, and medical characteristics of participants, non-participants, and patients who wanted no further contact with researchers. Values are numbers (percentages) unless indicated otherwise

\begin{tabular}{lccc} 
& $\begin{array}{c}\text { Participants } \\
(\mathbf{n}=73)\end{array}$ & $\begin{array}{c}\text { Non-participants } \\
(\mathbf{n}=\mathbf{8 1})\end{array}$ & $\begin{array}{c}\text { No contact } \\
(\mathbf{n}=30)\end{array}$ \\
\hline Age 18-39 & $62(85)$ & $59(73)$ & $25(83)$ \\
\hline Age $\geqslant 40$ & $11(15)$ & $22(27)$ & $5(17)$ \\
\hline Median (range) distance from study site $(\mathrm{km}):$ & $33.8(2-800)$ & $46.7(2-694)$ & $66(2-496)$ \\
\hline Single & $22(30)$ & $27(33)$ & $13(43)$ \\
\hline Married or cohabiting & $46(63)$ & $53(65)$ & $11(37)$ \\
\hline Separated or divorced & $5(7)$ & $1(1)$ & $6(20)$ \\
\hline No of children & $43(59)$ & $43(53)$ & $17(57)$ \\
\hline$\geqslant 1$ child & $30(41)$ & $38(47)$ & $13(43)$ \\
\hline Social class: & & & $1(4)$ \\
\hline I (Professional, managerial) & $10(14)$ & $4(5)$ & $6(23)$ \\
\hline II (Professional, managerial) & $23(32)$ & $28(35)$ & $10(38)$ \\
\hline III (Manual, non-manual, non-managerial) & $25(34)$ & $31(38)$ & $5(19)$ \\
\hline IV and V (Manual, labourers) & $5(7)$ & $7(9)$ & $4(15)$ \\
\hline Unemployed & $10(14)$ & $11(13)$ & 4 \\
\hline Not known & 0 & 0 & NR \\
\hline Educational qualifications: & $12(16)$ & $11(14)$ & NR \\
\hline None & $21(29)$ & $30(38)$ & NR \\
\hline O and A level & $40(55)$ & $38(48)$ & NR \\
\hline Degree, diploma, or postgraduate qualification & 0 & 2 & \\
\hline Not known & $30(41)$ & $33(43)$ & \\
\hline Has a religious affiliation: & $43(59)$ & $43(57)$ & 5 \\
\hline No & 0 & & \\
\hline Yes & & & \\
\hline Not known & 0 & \\
\hline Do you & & & \\
\hline
\end{tabular}

Do you have any current psychological

problems?

\begin{tabular}{lccc}
\hline Yes (receiving treatment) & $5(7) \dagger$ & $4(5) \ddagger$ & NR \\
\hline Yes (not receiving treatment) & $9(13) \dagger$ & $1(1)$ & NR \\
\hline No & $58(81) \S$ & $73(94)$ & NR \\
\hline Not
\end{tabular}

Not known

Have you had psychological problems

previously?*:

\begin{tabular}{lccc}
\hline Yes (received treatment) & $9(13)$ & $6(8)$ & NR \\
\hline Yes (did not receive treatment) & $10(14)$ & $2(3)$ & NR \\
\hline No & $53(74)$ & $70(90)$ & NR \\
\hline Not known & 1 & 3 & \\
\hline Do you have someone to confide in?: & & & NR \\
\hline Yes & $59(81)$ & $70(89)$ & NR \\
\hline No & $14(19)$ & $9(11)$ &
\end{tabular}

Did you receive sufficient information from your general practitioner?:

\begin{tabular}{llll}
\multicolumn{2}{l}{ Did you receive sufficient information from your general practitioner?: } & & \\
\hline Yes & $46(63)$ & $50(68)$ & NR \\
\hline No & $27(37)$ & $24(32)$ & NR \\
\hline
\end{tabular}

Not known

\begin{tabular}{llll}
\hline Yes & $52(71)$ & $62(85)$ & NR \\
\hline No & $21(29)$ & $11(15)$ & NR \\
\hline
\end{tabular}

\begin{tabular}{lccc} 
No & $21(29)$ & $11(15)$ & NR \\
\hline Not known & 0 & 8
\end{tabular}

Did you receive sufficient information from the tertiary oncologist?:

\begin{tabular}{lccc}
\hline Yes & $71(97)$ & $74(100)$ & NR \\
\hline No & $2(3)$ & 0 & NR \\
\hline Not known & 0 & 7 & \\
\hline Do you use alternative medicine?: & $4(6)$ & $1(1)$ & NR \\
\hline Yes & $69(95)$ & $73(99)$ & NR \\
\hline No & 0 & 7 & NR \\
\hline Not known & & & \\
\hline Histology: & $35(48)$ & $34(42)$ & $12(40)$ \\
\hline Teratoma & $38(52)$ & $41(51)$ & $17(57)$ \\
\hline Seminoma & 0 & $6(7)$ & $1(3)$ \\
\hline Mixed & & & $29(97)$ \\
\hline Stage of disease ${ }^{\star \star *}$ & $35(48)$ & $57(71)$ & 0 \\
\hline Stage I & $23(32)$ & $17(21)$ & $1(3)$ \\
\hline Small volume & $15(21)$ & $7(9)$ & $16(53)$ \\
\hline Large or very large volume & & & $12(40)$ \\
\hline Primary treatment ${ }^{\star * *}$ & $9(12)$ & $31(38)$ & $2(7)$ \\
\hline Surveillance & $22(30)$ & $24(30)$ & \\
\hline Radiotherapy & $42(58)$ & $26(32)$ & \\
\hline Chemotherapy & & & \\
\hline
\end{tabular}

$N R=$ Data not recorded. ${ }^{*} \mathrm{P}<0.05,{ }^{* *} \mathrm{P}<0.01,{ }^{* * *} \mathrm{P}<0.001$ for participants $v$ non-participants plus no contact group if data recorded. †Includes 1 patient receiving psychotropic drugs. $\ddagger$ All 4 patients receiving psychotropic drugs. §Includes 3 patients receiving psychotropic drugs. 
Table 3 Comparison of scores on psychosocial scales of participants and non-participants at baseline and one year

\begin{tabular}{|c|c|c|c|c|c|c|}
\hline \multirow[b]{2}{*}{ Questionnaire } & \multirow[b]{2}{*}{$\begin{array}{l}\text { No of } \\
\text { patients }\end{array}$} & \multicolumn{4}{|c|}{ At baseline } & \multirow[b]{2}{*}{$\begin{array}{c}\text { At } 1 \text { year (difference in } \\
\text { means }(95 \% \mathrm{CI})) \neq\end{array}$} \\
\hline & & $\begin{array}{l}\text { Mean score } \\
\text { (SD) }\end{array}$ & Median & Range & $P$ value & \\
\hline \multicolumn{7}{|c|}{ Hospital anxiety and depression } \\
\hline Participants & 73 & $7.1(4.1)$ & 6 & $0-18$ & 0.039 & $-0.8(-2.1$ to 0.4$)$ \\
\hline Non-participants & 81 & $5.7(3.5)$ & 5 & $0-17$ & & \\
\hline Non-participants & 81 & $2.4(2.8)$ & 1 & $0-15$ & & \\
\hline \multicolumn{7}{|c|}{ Mental adjustment to cancer } \\
\hline \multicolumn{7}{|l|}{ Fighting spirit: } \\
\hline Participants & 73 & $50.8(4.7)$ & 51 & $37-61$ & 0.34 & $-0.2(-2.0$ to 1.6$)$ \\
\hline Non-participants & 81 & $51.5(5.6)$ & 52 & $37-62$ & & \\
\hline Participants & 73 & $21.7(4.9)$ & 22 & $11-33$ & 0.011 & $-1.1(-2.5$ to 0.3$)$ \\
\hline Non-participants & 81 & $19.8(4.0)$ & 20 & $10-30$ & & \\
\hline \multicolumn{7}{|l|}{ Fatalism: } \\
\hline Participants & 73 & $16.1(3.2)$ & 16 & $8-23$ & 0.60 & $-0.9(-2.1$ to 0.1$)$ \\
\hline Non-participants & 81 & $15.9(3.1)$ & 16 & $8-22$ & & \\
\hline \multicolumn{7}{|l|}{ Denial: } \\
\hline Participants & 73 & $1.6(0.8)$ & 1 & $1-3$ & 0.002 & $0.2(-0.1$ to 0.5$)$ \\
\hline Non-participants & 81 & $2.1(1.0)$ & 2 & $1-4$ & & \\
\hline \multicolumn{7}{|c|}{ Rotterdam symptom checklist } \\
\hline Psychological sympt & & & & & & \\
\hline Participants & 73 & $62.9(16.8)$ & 62 & $30-102$ & 0.036 & $-2.6(-6.9$ to 1.6$)$ \\
\hline Non-participants & 81 & $68.3(16.3)$ & 71 & $30-101$ & & \\
\hline Rieker sexual adjust & & & & & & \\
\hline Participants & 73 & $8.1(2.8)$ & 7 & $6-20$ & 0.028 & $0.0(-1.4$ to 1.4$)$ \\
\hline Non-participants & 81 & $7.8(4.2)$ & 6 & $6-30$ & & \\
\hline Psychosocial adjust & & & & & & \\
\hline Healthcare orientatio & & & & & & \\
\hline Participants & 73 & $48.6(9.4)$ & 48 & $29-63$ & 0.21 & $0.3(-0.3$ to 3.7$)$ \\
\hline Non-participants & 81 & $50.9(8.2)$ & 51 & $29-73$ & & \\
\hline Vocational environm & & & & & & \\
\hline Participants & 73 & $55.9(5.8)$ & 57 & $45-63$ & 0.020 & $2.9(0.7 \text { to } 5.1)^{\star}$ \\
\hline Non-participants & 81 & $58.4(4.4)$ & 58 & $45-70$ & & \\
\hline Domestic: & & & & & & \\
\hline Participants & 73 & $46.0(6.8)$ & 45 & $34-63$ & 0.016 & $-0.5(-3.4$ to 2.5$)$ \\
\hline Non-participants & 81 & $43.3(7.5)$ & 42 & $34-65$ & & \\
\hline Sexual relationships: & & & & & & \\
\hline Participants & 73 & $46.9(6.1)$ & 47 & $40-61$ & 0.95 & $0.1(-2.3$ to 2.6$)$ \\
\hline Non-participants & 81 & $47.5(7.6)$ & 47 & $40-72$ & & \\
\hline Extended family: & & & & & & \\
\hline Participants & 73 & $51.5(7.5)$ & 46 & $46-66$ & 0.46 & 1.0 (-1.9 to 3.9$)$ \\
\hline
\end{tabular}

${ }^{*} \mathrm{P}<0.05,{ }^{\star *} \mathrm{P}<0.01$. From Mann-Whitney $U$ test. $¥$ Negative values for differences between groups favour participants. 
Table 4 Comparison of scores on anxiety and depression subscales of hospital anxiety and depression scale. Patients with testicular cancer who had adjuvant psychological therapy were the treatment group and those who had standard medical care were the control group. Values are proportions (percentages) (95\% confidence intervals) or mean scores (95\% confidence intervals)

Anxiety subscale

Depression subscale

Proportion of patients with case morbidity (scores $\geqslant 8$ on subscale)

\begin{tabular}{|c|c|c|c|c|}
\hline \multicolumn{5}{|l|}{ At baseline: } \\
\hline Treatment group & $12 / 36$ & $33 \quad$ (19 to 51$)$ & $6 / 36$ & $17 \quad$ (6 to 33$)$ \\
\hline Control group & $19 / 37$ & $51 \quad$ (34 to 68) & $5 / 37$ & $14 \quad$ (5 to 29$)$ \\
\hline \multicolumn{5}{|l|}{ At 2 months: } \\
\hline Treatment group & $5 / 35$ & $14 \quad(5$ to 30$)$ & $3 / 35$ & $9 \quad$ (2 to 23$)$ \\
\hline Control group & $16 / 36$ & $44 \quad$ (28 to 62) & $7 / 36$ & $19 \quad(8$ to 36$)$ \\
\hline \multicolumn{5}{|l|}{ At 4 months: } \\
\hline \multicolumn{5}{|l|}{ At 12 months: } \\
\hline Treatment group & $11 / 33$ & $33 \quad$ (18 to 52$)$ & $1 / 33$ & $3 \quad$ (1 to 15$)$ \\
\hline Control group & $10 / 35$ & $29 \quad(15$ to 46$)$ & $0 / 35$ & $0 \quad(0$ to 10$)$ \\
\hline \multicolumn{5}{|c|}{ Mean change from baseline scores } \\
\hline \multicolumn{5}{|l|}{ At 2 months: } \\
\hline \multicolumn{5}{|l|}{ At 4 months: } \\
\hline Treatment group & & $-1.34(-2.52$ to -0.17$)$ & & $-0.46(-1.64$ to 0.72$)$ \\
\hline Control group & & $-1.74(-2.93$ to -0.56$)$ & & $-0.80(-2.01$ to 0.41$)$ \\
\hline Treatment effect† & & $0.40(-1.24$ to 2.04$)$ & & $0.34(-1.31$ to 2.00$)$ \\
\hline \multicolumn{5}{|l|}{ At 12 months: } \\
\hline Treatment group & & $-0.97(-2.24$ to 0.30$)$ & & $-0.97(-1.95$ to 0.02$)$ \\
\hline Control group & & $-2.63(-4.00$ to $(-1.25)$ & & $-2.00(-3.35$ to -0.65$)$ \\
\hline Treatment effect† & & $1.66\left(-0.18\right.$ to $\left.3.50^{*}\right)$ & & $1.03(-0.63$ to 2.69$)$ \\
\hline
\end{tabular}

${ }^{\star} P<0.05$. †Difference in means between groups; negative value favours treatment group.

\section{Baseline characteristics of treatment and control} groups

At baseline there were no significant differences in psychosocial variables between participants randomly allocated to adjuvant psychological therapy or standard treatment (data not shown). However, though not conventionally significant, there were differences between the groups in histology and stage of disease. Twenty one patients in the treatment group were diagnosed with teratoma compared with 13 in the control group, and 22 patients in the treatment group had stage I disease compared with 13 in the control group.

\section{Evaluation of adjuvant psychological therapy}

At the 2 month assessment the median number of psychotherapy sessions received was 3 (range 1-13). Altogether, 21 patients received additional sessions after 2 months; the median number of sessions received at 12 months was 4 (range 1-16). The end of therapy was mainly determined by patient and therapist together.

Comparison with baseline scores generally suggested improvements for both the treatment and control groups at all points. However, at 2 months worse scores were found in those subscales influenced by physical factors (data not shown).

Although adjuvant psychological therapy did not seem to be effective, the treatment group showed a marginal reduction in anxiety at two months (mean difference between groups - 1.41 (95\% confidence interval -2.86 to $0.03, \mathrm{P}=0.03$, Mann-Whitney $\mathrm{U}$ test). When adjusted for histology, stage of disease, and type of treatment planned the effect was not significant. No difference between groups was observed for depression scores $(-0.51(-2.18$ to 1.15$))$. By one year the lack of benefit of adjuvant psychological therapy was apparent; control patients seemed to fare better than treated patients (difference in anxiety subscale score of the hospital anxiety and depression scale $1.66(-0.18$ to 3.50$), \quad \mathrm{P}=0.03$, Mann-Whitney $\mathrm{U}$ test). The proportion of patients scoring above the threshold on subscales of the hospital anxiety and depression scale was not influenced by adjuvant psychological therapy.

\section{Discussion}

Participation in the trial was not restricted to those with high psychosocial morbidity scores, which implies that formal dysfunction scores may not be indicators of a need for support or of issues relevant to patients with testicular cancer. Men with cancer may be anticipating problems related to work and fear of having a relapse, ${ }^{9}$ which may warrant an educational rather than a therapeutic approach to intervention. Our study found that, consistent with other studies, ${ }^{40}$ participants had higher scores than non-participants on measures of dysfunction at baseline. This suggests that the minority who agreed to participate did so because they felt in need of psychological support.

Sixty per cent of eligible men declined to participate in the trial. Randomisation was not cited as a reason for declining to participate. Patients with stage I disease were most likely to refuse to participate; in addition to a particularly reassuring prognosis, these patients receive outpatient follow up at our hospital and may not have perceived adjuvant psychological therapy as part of their treatment. Inpatients not only were feeling worse physically but may have agreed to participate since participation did not require a special journey or taking time out from work. If counselling is 
to be offered to patients with testicular cancer, it should be part of a coordinated package of care designed to minimise social disruption.

\section{Adjuvant psychological therapy}

No clinically important differences occurred in patients who attended adjuvant psychological therapy. Because numbers in this study were small, only large differences between treatment groups could be detected. The only apparent benefit was measured on the anxiety subscale at 2 months, when patients undergoing aggressive treatment may need supportive care in addition to routine management. All scores for both groups were generally below the threshold for identification of cases. The treatment group, however, was no better off than the control group in terms of anxiety at one year. Reasons for this are unclear; an inappropriate intervention cannot be discounted nor can the effect of a therapist. The therapist might have had a beneficial or an adverse effect on the patients, but we could not control for this since we had resources for only one therapist. Alternatively, treated patients may have been more able to acknowledge their negative feelings. We do not know the extent to which the patients may have relinquished their sense of masculinity as a result of agreeing to psychological counselling which might possibly induce or prolong anxiety. However, this may have been beneficial in allowing men to lower their psychological defences in the face of chaos and to experience an inner connection.

There was considerable variability in the number of therapy sessions attended. A few patients required only a single treatment consisting of reassurance and information. Attendance at fewer than six of the planned sessions did not necessarily indicate non-compliance by the patient but may have been the result of an agreement between the therapist and the patient to end therapy. Thirteen sessions were attended by one highly anxious patient by the assessment at 8 weeks; altogether he attended 16 sessions over 4 months until his chemotherapy regimen ended. Another patient attended three sessions by the 8 week assessment; after this his disease progressed and he died having attended a total of 10 sessions between the assessments at 4 and 12 months. Many patients may require support but men with high anxiety scores who receive aggressive chemotherapy may require more intensive intervention. Psychotherapeutic support may have been no more or less effective than the standard care received by patients in the testicular tumour unit in our hospital, and this may account for the homogeneity of outcome both between those in the treatment group and those in the control group and between participants and non-participants.

One concern about offering psychotherapy to patients with cancer is that those who refuse psychotherapy may have hidden morbidity and thus they may have unmet needs for psychological intervention. Our results show that such morbidity does not exist or that it is not identified by the instruments in general use. Non-participants seemed to have adjusted well over the year studied.
Key messages

- Counselling for patients with cancer is widely advocated, although its effectiveness has not been fully evaluated

- No study of patients with cancer has evaluated a psychological intervention in young men or in a group of patients with a disease with an excellent prognosis

- Most patients with testicular cancer declined to participate in this randomised trial of adjuvant psychological therapy, and those who participated had more psychosocial dysfunction

- No evidence of benefit was observed after treatment with adjuvant psychological therapy in this group of patients

- There were no consistently significant differences in psychosocial outcome over one year between those who agreed to participate and those who declined to participate

\section{Completion of questionnaires}

Studies that evaluate psychosocial outcome using questionnaires completed by participants are prone to problems of non-completion. In this study compliance with assessment completion was very high within the randomised trial; only one patient did not complete questionnaires at two of the three assessment points (figure). Four patients died during the trial and two additional individual assessments were not completed because the patient was too ill. Even if these patients had had scores at the extremes of those reported for the group, their omission is unlikely to have introduced significant bias into the evaluation of treatment efficacy. More of the patients who declined randomisation but agreed to complete questionnaires (nonparticipants) did not complete their later assessments. Most of these patients were well, and the omission of their assessments is unlikely to have seriously influenced the comparison. The study coordinator (CM) remained blind to patient allocation in all except two instances throughout follow up. Her influence is unlikely to have confounded the comparison between treatment groups.

\section{Need for caution}

This study shows that there is a need for caution. Counselling is often presented as an integral part of sound medical practice ${ }^{1}$; ironically, this may be creating a split between the care of mind and body by healthcare professionals, who may think that their duties of informing and reassuring patients should be passed to a counsellor. Generalisations of treatment efficacy across different types of patient groups should be avoided. The efficacy of different psychotherapeutic interventions is likely to differ according to the type of disease, and interventions must be systematically evaluated in each specific context. In the United Kingdom the current trend for providing counselling for patients with cancer may conflict with the need of healthcare purchasers to direct resources to where they can be of greatest benefit. ${ }^{21}$

We thank Ros Gardner, Sandy McVeigh, and the nurses in outpatient clinics and inpatient wards who facilitated the research 
and helped with its technical aspects, and also the patients who took part in this study.

Funding: This study was funded by the Cancer Research Campaign and the Bob Champion Cancer Trust. Conflict of interest: None.

1 Davis H, Fallowfield L. Counselling and communication in health care. 4th ed. Chichester: Wiley, 1991.

2 Spiegel D, Bloom JR, Yalom I. Group support for patients with metastatic cancer. Arch Gen Psychiatry 1981;38:527-33.

3 Fawzy I, Cousins N, Fawzy NW, Kemeny ME, Elashoff R, Morton D. A structured psychiatric intervention for cancer patients. Arch Gen Psychiatry 1990;47:720-5.

4 Greer S, Moorey S, Baruch JDR, Watson M, Robertson BM, Mason A, et al. Adjuvant psychological therapy for patients with cancer: a prospective randomised trial. BMJ 1992;304:675-80.

5 Moorey S, Greer S. Adjuvant psychological therapy for patients with cancer: outcome at one year. Psycho-Oncology 1994;3:39-46.

6 Bloom JR, Ross RD, Burnell G. The effect of social support on patient adjustment after breast surgery. Patient Counselling Health Educ 1978; Autumn:50-9.

7 Golonka LM. The use of group counselling with breast cancer patients receiving chemotherapy. Dissertation Abstracts International 1977;37: 6362-3.

8 Moynihan C. Psychosocial assessments and counselling. In: Horwich A, ed. Testicular cancer: investigation and management. London: Chapman and Hall, 1996.

9 Moynihan C. Testicular cancer: the psychosocial problems of patients and their relatives. Cancer Surv 1987;6:477-510.

10 Rieker PP, Fitzgerald EM, Kalish LA, Richie JP, Lederman GS, Edbril SD, et al. Psychosocial factors, curative therapies and behavioural outcomes: a comparison of testis cancer survivors and a control group of healthy men. Cancer 1989;64:2399-406.

11 House A, Knapp P. Trials that use Zelen's procedure should be acceptable. BMJ 1997:315:251.
12 Vingerhoets A, van Heck G. Gender, coping and psychosomatic symptoms. Psychol Med 1990;20:125-35.

13 Moorey S, Greer S. Psychological therapy for patients with cancer: a new approach. Oxford: Heinemann Medical, 1989.

14 Zigmond AS, Snaith RP. The hospital anxiety and depression scale. Acta Psychiatr Scand 1983;67:361-70.

15 Greer S, Watson M. Mental adjustment to cancer: its measurement and prognostic importance. Cancer Surv 1987;6:439-53.

16 Derogatis LR. Psychosocial adjustment to illness scale (PAIS-SR). Baltimore, MD: Clinical Psychometric Research, 1983

17 De Haes JC, Knippenberg FC, Nejit JP. Measuring psychological and physical distress in cancer patients: structure and application of the Rotterdam symptom checklist. Br J Cancer 1990;62:1034-8.

18 Brannon R, Juni S. A scale for measuring attitudes about masculinity. Psychol Doc 1984;14:2612.

19 Wing JK, Cooper JE, Sartorius N. The measurement and classification of psychiatric symptoms. Cambridge: Cambridge University Press, 1980.

20 Bauman LJ, Gervey R, Siegel K. Factors associated with cancer patients' participation in support groups. J Psychosoc Oncol 1992;10(3):1-20.

21 Fahy T, Wessely S. Should purchasers pay for psychotherapy? BMJ 1993;307:576-7.

(Accepted 22 October 1997)

\section{Correction}

Randomised controlled trial to evaluate early discharge scheme for patients with stroke

An authors' error occurred in this paper by Anthony G Rudd et al (25 October, pp 1039-44). In table 3 the $P$ value for the hospital anxiety and depression scale should have read 0.06 rather than 0.02 .

\title{
Evaluation of reagent strips in detecting asymptomatic bacteriuria in early pregnancy: prospective case series
}

\author{
Douglas G Tincello, David H Richmond
}

\begin{abstract}
Objective: To evaluate the performance of reagent test strips in screening pregnant women for asymptomatic bacteriuria at their first visit to an antenatal clinic.

Design: Prospective case series.

Setting: Antenatal clinic of a large inner city maternity hospital.

Subjects: All women attending for their first antenatal clinic. Patients taking antibiotics for any reason and those with urinary tract symptoms were excluded. Intervention: A midstream urine specimen was divided; half was sent for microscopy and formal bacteriological culture and the other half was tested with a commercial reagent strip test for the presence of blood, protein, nitrite, and leucocyte esterase.

Main outcome measures: Sensitivity, specificity, and positive and negative predictive values of the reagent strips in diagnosing asymptomatic bacteriuria (defined as $10^{5}$ colony forming units $/ \mathrm{ml}$ urine).

Results: Sensitivity was low, with a maximum of 33\% when all four tests were used in combination. Specificity was high, with typical values of $99 \%$ or more. Positive predictive value reached a maximum of $69 \%$ and negative predictive value was typically $95 \%$ or more.
\end{abstract}

Conclusion: Urine reagent strips are not sufficiently sensitive to be of use in the screening for asymptomatic bacteriuria and therefore many patients would be missed. In view of the potentially serious sequelae of this condition in pregnant women we recommend that formal bacteriological investigation remain the investigation of choice in this group of patients.

\section{Introduction}

Reagent strip testing of urine specimens for infection has become widespread in many areas of clinical practice. Such strips were sensitive and specific when used to exclude urinary tract infection in patients attending a urodynamic clinic ${ }^{1}$ and in pregnant women with symptoms of urinary tract infection. ${ }^{2}$ These reagent strips can undoubtedly realise substantial cost savings by reducing the need to send urine specimens for formal microscopy and culture, ${ }^{2-4}$ but none of the published studies has examined the performance of reagent test strips as a screening test for asymptomatic bacteriuria exclusively in women attending their first antenatal clinic.

Asymptomatic bacteriuria is defined as a pure culture of at least $10^{5}$ organisms $/ \mathrm{ml}$ of urine. ${ }^{5}$ Detection of all women with asymptomatic bacteriuria is important as $25 \%$ to $30 \%$ will develop symptomatic urinary tract infection while pregnant. ${ }^{6}$ The sequelae of urinary tract infection in pregnancy include pyelonephritis, premature labour, and preterm rupture of the membranes. $^{578}$ In view of the potential importance of
Department of Urogynaecology, Liverpool Women's Hospital, Liverpool L8 7SS

Douglas G Tincello, research registrar David H Richmond, consultant gynaecologist

Correspondence to: Dr Tincello

BMJ 1998;316:435-7 